\title{
Safe handling of food at home or cottage
}

Background and epidemiology: In Canada, summertime and eating home-prepared foods, especially meats, are risk factors for contracting an enteric illness. Almost half (47\%) of the 44451 laboratory-confirmed sporadic cases of gastroenteritis caused by any of 8 enteric pathogens (listed, with their incidences, in Table 1) reported to public health units in Ontario between 1997 and 2001 occurred during the 4-month period from June through September. ${ }^{1}$ The most common foods involved were poultry and other meat items (68\%). The associated settings were primarily private homes $(50 \%)$, followed by travel $(25 \%)$ and restaurants (14\%). Public health efforts to reduce the incidence of these illnesses need to be directed at improving safe food handling practices at home, especially during the warm months. ${ }^{1}$

To identify risky food-preparation behaviours and the populations that practise them, in 1995 the US Centers for Disease Control and Prevention, Food and Drug Administration and several state health departments administered a food safety questionnaire: the state-based Behavioral Risk Factor Surveillance System ${ }^{2}$ surveyed noninstitutionalized adults about their health behaviours by means of randomized telephone dialing.

Table 1: The 8 most commonly reported enteric pathogens in Ontario, 1997-2001

\begin{tabular}{lc}
\hline Pathogen & Incidence* \\
\hline Campylobacter & 42.3 \\
Salmonella & 22.6 \\
Verotoxin-producing & \\
Escherichia coli & 3.7 \\
Yersinia & 3.0 \\
Shigella & 2.7 \\
Hepatitis A & 2.3 \\
Listeria & 0.3 \\
Clostridium botulinum & $<0.3$ \\
\hline *Average annual incidence per 100000 \\
population.
\end{tabular}

They found that a high proportion of the 19356 respondents reported risky ways of handling food during the preceding 12 months (Table 2).

High-risk food-handling behaviours were more common in specific populations. Men (24\%) were more likely than women $(15 \%)$ to eat pink hamburger and, among those who remembered seeing safe food-handling information on labels, were less likely ( $68 \%$ of men v. $82 \%$ of women) to read it. Age, education and income also influenced risky behaviour, with consumption of pink hamburger, for example, decreasing in prevalence with increasing age, yet (somewhat paradoxically) increasing with levels of education and income. ${ }^{2}$

Clinical management: In addition to providing care to the individual, the role of the primary care physician includes assessing the public health implications of each case of gastroenteritis. A stepwise approach to patient management involves gauging the severity of the illness, managing fluids and electrolytes if necessary, alleviating symptoms when appropriate, narrowing the differential diagnosis, confirming the cause, deciding about antimicrobial treatment (which is rarely indicated) and preventing additional cases. ${ }^{3}$ Certain populations, such as people who are elderly, very young or immunocompromised, are vulnerable to severe illness. Detection and control of an outbreak can begin in a physician's office with a careful history of exposure (including enquiries about whether the patient's occupation involves handling food), a request for a stool sample and a call to the local health unit. Early detection can save lives.

Prevention: Laboratory personnel notify the public health unit of confirmed cases; staff at the health unit follow each case to determine if it is sporadic or part of a larger outbreak. This process usually involves providing education about safe foodhandling techniques. Such information is often available from the local health unit and is widely available on the Internet (e.g., at www.porcupinehu.on.ca /inspection/safe.html). In a nutshell, the advice is "CSCC": Clean (wash hands and surfaces often), Separate (avoid crosscontamination), Cook (heat foods to a proper temperature) and Chill (refrigerate promptly).

\section{Erica Weir \\ CMAF \\ References \\ 1. Lee MB, Middleton D. Enteric illness in Ontario, Canada, from 1997 to 2001. 7 Food Prot 2003;66(6):953-61. \\ 2. Yang S, Leff MG, McTague D, Hor- vath K, Jackson-Thompson J, Murayi $\mathrm{T}$, et al. Multistate surveillance for food handling, preparation and con- sumption behaviors associated with foodborne diseases: 1995 and 1996 BRFSS food-safety questions. $M M W R$ CDC Surveill Summ 1998;47(4):33-57. \\ 3. Townes JM. Acute infectious gastro- enteritis in adults: seven steps to man- agement and prevention [review]. Postgrad Med 2004;115(5):11-6.}

Table 2: Results of a 1995 food safety survey ${ }^{2}$

\begin{tabular}{lc}
\hline Behaviour & $\begin{array}{c}\% \text { of } 19356 \text { respondents } \\
(95 \% \text { confidence interval) }\end{array}$ \\
\hline Eating undercooked eggs & $50.2(49.2-51.2)$ \\
Eating pink hamburger & $19.7(18.9-20.5)$ \\
Eating raw oysters & $8.0(7.5-8.5)$ \\
Drinking raw, unpasteurized milk & $1.4(1.2-1.6)$ \\
Not washing hands with soap after & \\
handling raw meat & $18.6(17.8-19.4)$ \\
Not washing cutting board with soap & \\
or bleach after putting raw meat on it & $19.5(18.6-20.4)$ \\
\hline
\end{tabular}

